\title{
Early Intratracheal Administration of Corticosteroid and Pulmonary Surfactant for Preventing Bronchopulmonary Dysplasia in Preterm Infants with Neonatal Respiratory Distress Syndrome: A Meta-analysis
}

\author{
Yan-yan ZHONG ${ }^{1}$, Jin-chun $\mathrm{LI}^{2}$, Ya-ling $\mathrm{LIU}^{3}$, Xiao-bo ZHAO ${ }^{3}$, Musa MALE${ }^{4}$, Dong-kui $\mathrm{SONG}^{3}$, Yan BAI ${ }^{3 \#}$ \\ ${ }^{1}$ Department of Pediatrics, Huazhong University of Science and Technology Hospital, Wuhan 430074, China \\ ${ }^{2}$ Neonatal Intensive Care Unit, Hubei Maternity and Children's Hospital, Wuhan 430070, China \\ ${ }^{3}$ Department of Pediatrics, Union Hospital, Tongji Medical College, Huazhong University of Science and Technology, Wuhan \\ 430022, China \\ ${ }^{4}$ Department of Urology, Tongji Hospital, Tongji Medical College, Huazhong University of Science and Technology, Wuhan \\ 430030, China
}

(C) Huazhong University of Science and Technology 2019

\begin{abstract}
Summary: There is uncertain result with regard to the use of inhalation or instillation steroids to prevent bronchopulmonary dysplasia in preterm infants. This meta-analysis was designed to evaluate the efficacy and safety of early airway administration (within 2 days after birth) of corticosteroids and pulmonary surfactant (PS) for preventing bronchopulmonary dysplasia (BPD) in premature infants with neonatal respiratory distress syndrome (NRDS). The related studies were retrieved in PubMed, EMBASE, the Cochrane Library, Clinical Trial, CNKI, Wanfang and VIP Database from inception to August 2018. Two reviewers independently screened the studies to ensure that all patients with diagnosis of NRDS were enrolled to studies within 1 day after birth, assessed the quality of included studies by GRADEpro system and extracted the data for review. The meta-analysis was performed by RevMan 5.2 software. A subgroup analysis about inhaled corticosteroid (ICS) delivery method was made between ICS inhalation subgroup [inhalation of ICS by nebulizer or metered dose inhaler (MDI)] and ICS intratracheal instillation subgroup (PS used as a vehicle). Eight randomized controlled trials were enrolled in the meta-analysis, 5 trials of which stated the randomized method, grouping and blinded method, and the follow-up procedures were reported. GRADEpro system showed high quality of 4 trials (5 articles), and the rest 4 trials had moderate quality. Meta-analysis showed that the incidence of BPD was decreased in ICS group, the relative risk (RR) was 0.56 (95\% CI: $0.42-0.76)$, and similar trends were found in ICS inhalation subgroup and ICS intratracheal instillation subgroup, with the corresponding RR being 0.58 (95\% CI: $0.41-0.82)$ and 0.47 (95\% CI: $0.24-0.95)$ respectively. ICS could also significantly reduce the mortality risk as compared with placebo control group (RR: 0.67; 95\% CI: 0.45-0.99), with RR of ICS inhalation subgroup and ICS intratracheal instillation subgroup being 0.81 (95\% CI: $0.34-1.94)$ and 0.64 (95\% CI: $0.41-0.99)$ respectively. Moreover, the percentage of infants using PS more than one time was lower in ICS group than in the placebo control group, with the RR and 95\% CI being 0.55 (95\% CI: 0.45-0.67), and that in ICS intratracheal instillation subgroup lower than in ICS inhalation subgroup (RR: 0.56 ; $95 \%$ CI: $0.45-0.69$, and RR: 0.35 ; $95 \%$ CI: $0.08-1.52$ respectively). There was no significant difference in the incidence of infection or retinopathy of prematurity and neuro-motor system impairment between ICS group and placebo control group, with the corresponding RR being 0.95 (95\% CI: $0.59-1.52), 0.92$ (95\% CI: $0.62-1.38$ ) and 1.13 (95\% CI: 0.92-1.39), respectively. It was concluded that early administration of ICS and PS is an effective and safe option for preterm infants with NRDS in preventing BPD and reducing mortality, decreasing the additional PS usage, especially for the ICS intratracheal instillation subgroup. Furthermore, the appropriate dose and duration of ICS, combined use of inhalation or instillation of ICS with PS and the long-term safety of airway administration of corticosteroids need to be assessed in large trials.
\end{abstract}

Key words: corticosteroid; pulmonary surfactant; preterm infants; bronchopulmonary dysplasia; neonatal respiratory distress syndrome; meta-analysis

Yan-yan ZHONG, E-mail: zhongyy0629@qq.com

${ }^{\#}$ Corresponding author, E-mail: yanbaixh@hust.edu.cn 
Neonatal respiratory distress syndrome (NRDS) is a common respiratory disease in premature infants. For premature infants with severe NRDS after longterm mechanical ventilation treatment, the damage by mechanical ventilation and high concentration oxygen can result in the lung tissue structure reconstruction, showing tissue fibrosis, thickened basement membrane of bronchi and capillaries, and alveoli apoptosis under the airway biopsy. These pathological changes of airway can reduce pulmonary ventilation function, increase ventilation resistance, and prolong ventilation time. Those infants with severe NRDS are at high risk of bronchopulmonary dysplasia (BPD) ${ }^{[1]}$, which increases the risk of neonatal death, and the risk of future growth retardation and impaired lung function in surviving children. Therefore, it is very important to prevent the occurrence of BPD. Various evidence indicates that pulmonary inflammation plays an important role in the pathogenesis of BPD. Glucocorticoids suppress the lung inflammation and have been used to treat or prevent $\mathrm{BPD}^{[2-4]}$ for more than a decade; however, because of the systemic adverse effects, glucocorticoids are not recommended for routine use $\mathrm{e}^{[5]}$. Nevertheless, the local beneficial effects of systemic glucocorticoid on the respiratory tract are well documented $\mathrm{d}^{[6-8]}$. One alternative to systemic administration is delivery of glucocorticoids by inhalation ${ }^{[9,10]}$ or by intratracheal instillation. Pulmonary surfactant (PS) is now routinely used in NRDS. After PS instillation, there is a rapid distribution to the lung periphery ${ }^{[1,}{ }^{12]}$, probably resulting from a dragging force created by surface tension gradient (Marangoni effect) between the instilled surfactant and the airway liquid ${ }^{[13]}$. Marangoni effect would facilitate the instilled medication, such as steroid or antibiotics, in reaching the periphery of the lungs ${ }^{[14,15]}$. Several trials had investigated the effects of the intra-tracheal application of ICS and PS on the incidence of BPD and mortality, but varied on the study population, therapy intervention and therapy initial time and duration. This meta-analysis was designed to enroll high quality studies, assess the impact of early airway administration of ICS and PS on the incidence of BPD in premature infants with NRDS, and provide a reference for clinical applications.

\section{MATERIALS AND METHODS}

\subsection{Eligibility Criteria}

Enrolled studies had to meet the following criteria: (1) study subjects were premature infants, with gestational age less than 36 weeks, and the diagnosis of NRDS was confirmed; (2) infants were randomized to receive treatment with airway administration (inhalation or instillation) of corticosteroid and PS (ICS group) or placebo plus PS (placebo control group); (3) interventions started within 1 day after birth; (4) more than one of the outcomes was reported. The exclusion criteria were as follows: (1) non-clinical studies, observational or retrospective studies, or therapeutic studies; (2) duplicate reports or secondary or post-hoc analyses of the same study population; (3) lack of sufficient information on baseline or primary or secondary outcome data.

\subsection{Outcome Definition}

Following outcomes were evaluated in this study, including: (1) BPD incidence, (2) mortality, (3) percentage of infants using PS more than one time, (4) infection (sepsis) incidence, (5) premature retinopathy (ROP) incidence, (6) incidence of neurological lesions. The item (1) was defined as the primary outcome, and items (2)-(6) as the secondary outcomes.

\subsection{Search Strategy of the Enrolled Studies}

The study searches were carried out in multiple databases, including PubMed, Web of Science, Embase, Cochrane Library, Clinicaltrials.gov, Controlled-trials. com, Google scholar, VIP, WanFang and proceedings of the Pediatric Academic Society meetings from database from inception to August 2018. Search terms included: preterm infant, premature infant, bronchopulmonary dysplasia, chronic lung diseases, anti-inflammatory agents, neurodevelopmental outcomes, steroids, glucocorticoids, corticosteroids, budesonide, beclomethasone, flunisolide, and fluticasone. No language restriction was applied.

\subsection{Study Screening, Quality Evaluation, and Data Extraction}

Two reviewers independently conducted study screening, quality evaluation, data extraction and crosscheck. All discrepancies were resolved by discussion and consensus. After reading the title and abstracts to exclude studies that were not related to the topic, the full-text was reviewed to decide whether to include or not. Quality and bias risk of studies were assessed by GRADEpro system. For each study, data were extracted independently using a predesigned form. Any differences and disagreements in the abstracted data were discussed and resolved by consensus. Details of study design, analysis, and results were abstracted.

\subsection{Statistical Methods}

Meta-analysis was performed by RevMan 5.2 software. When there was no statistical heterogeneity $\left(I^{2}<50 \%\right)$, the heterogeneity test was performed using a fixed-effect model; otherwise, if there was statistical heterogeneity $\left(I^{2} \geq 50 \%\right)$, random-effect model was used. Treatment effect estimates for all trials were calculated, expressed as typical relative risk (RR) for discontinuous outcomes and weighted mean difference (WMD) for continuous outcomes, all with a $95 \%$ confidence interval (CI). $P$ values of $<0.05$ and RR estimate $95 \%$ CI $<1.00$ or $>1.00$ were considered statistically significant. Subgroup analysis would be executed if there were different deliver approaches of ICS. 


\section{RESULTS}

\subsection{Study Selection}

168 potential trials were identified during the initial study search, of which 15 RCTs involved the application of corticosteroid in premature infants with NRDS. Fig. 1 shows the details of the selection process. Eight ( 9 articles) of 15 studies presented early use of corticosteroid and sufficient data for further analysis ${ }^{[16-24]}$. 792 preterm infants were enrolled, including 414 patients receiving airway administration (inhalation or instillation) of corticosteroid and PS (ICS group) and 378 patients given placebo plus PS (placebo control group). The basic information of selected studies is shown in table 1.

\subsection{Study Quality and Bias Evaluation}

Eight studies were stated as RCTs, 5 of which described the implementation of random sequence generation procedure and allocation concealment, 4 of which were blinded to the treatment practitioners, which described shedding and loss of patients followup. Quality and bias evaluation of selected studies by GRADEpro system is shown in table 2.

\subsection{Meta-Analysis Results}

2.3.1 Summary of Findings The results are shown in table 3.

2.3.2 BPD Incidence Eight studies enrolling 838 patients reported the incidence of BPD. The studies on ICS subgroups showed heterogeneous, and the metaanalysis of the random effects model indicated that the ICS group was associated with a lower likelihood of BPD development than the placebo control group ( $R R=0.56 ; 95 \%$ CI: $0.42-0.76, P=0.0001$ ). Subgroup analysis based on delivery method of ICS showed

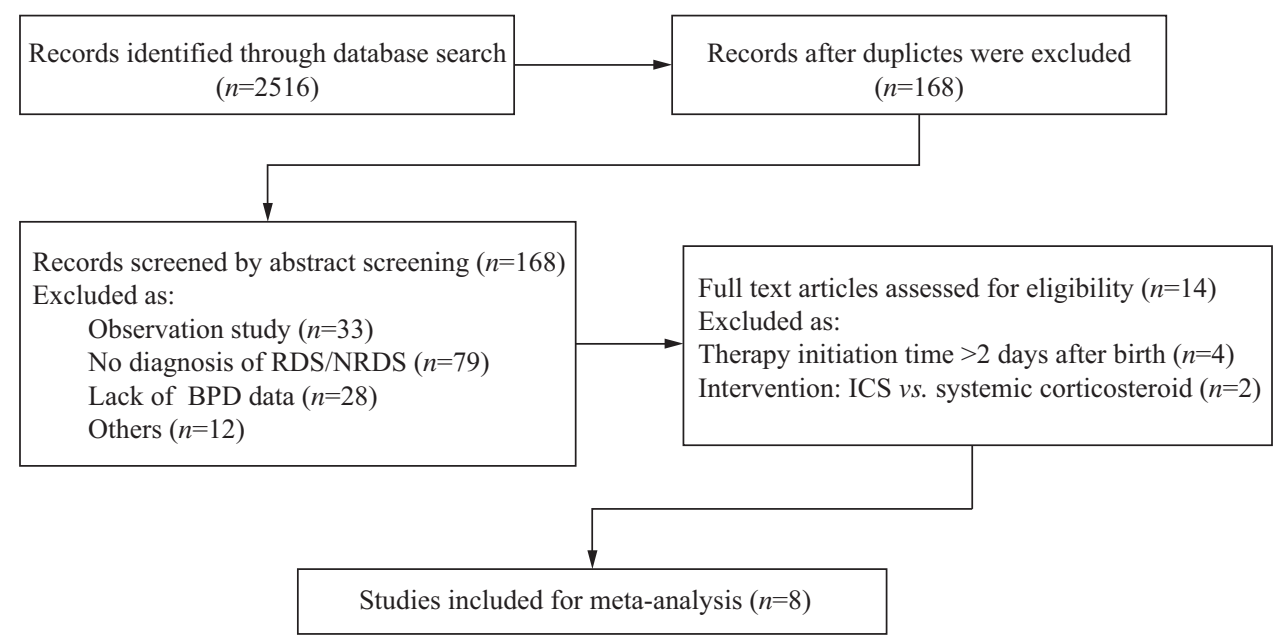

Fig. 1 Flow chart of the studies selection process

Table 1 Basic information of selected studies

\begin{tabular}{|c|c|c|c|c|c|c|}
\hline \multirow{2}{*}{ Study } & \multirow{2}{*}{ Enrollment criteria } & \multirow{2}{*}{$\begin{array}{l}\text { Time to start } \\
\text { intervention }\end{array}$} & \multicolumn{2}{|l|}{ Treatment } & \multicolumn{2}{|c|}{$n$} \\
\hline & & & ICS group & Placebo control group & ICS & Control \\
\hline $\begin{array}{l}\text { Kuo, } 2010^{[16]} \\
\text { Yeh, } 2008^{[17]}\end{array}$ & $\mathrm{BW}<1500 \mathrm{~g}, \mathrm{RDS}$ & $6 \mathrm{~h}$ after birth & BUD $0.25 \mathrm{mg} / \mathrm{kg}+\mathrm{PS} 100 \mathrm{mg} / \mathrm{kg}$ ia & PS $100 \mathrm{mg} / \mathrm{kg}$ ia & 60 & 60 \\
\hline $\begin{array}{l}\text { Sadeghnia, } \\
2018^{[18]}\end{array}$ & $\mathrm{GA}<28 \mathrm{w}, \mathrm{RDS}$ & $2 \mathrm{~h}$ after birth & BUD $0.5 \mathrm{mg}$ (NEB, bid until day 7) + PS ia & PS ia & 35 & 35 \\
\hline Yeh, 2016 $6^{[19]}$ & $\mathrm{BW}<1500 \mathrm{~g}, \mathrm{RDS}$ & $6 \mathrm{~h}$ after birth & BUD $0.25 \mathrm{mg} / \mathrm{kg}+$ PS $100 \mathrm{mg} / \mathrm{kg}$ ia & PS $100 \mathrm{mg} / \mathrm{kg}$ ia & 131 & 134 \\
\hline $\begin{array}{l}\text { Zimmeman, } \\
2000^{[20]}\end{array}$ & $\mathrm{BW}<1300 \mathrm{~g}, \mathrm{RDS}$ & $3 \mathrm{~h}$ after birth & BDP (pMDI 1-4/day for 12 days + PS ia) & $\begin{array}{l}\text { Placebo pMDI + } \\
\text { PS ia }\end{array}$ & 23 & 24 \\
\hline Cao, $2018^{[21]}$ & $\begin{array}{l}\mathrm{GA}<32 \mathrm{w}, \mathrm{RDS} \text { needing } \\
\text { ventilator support }\end{array}$ & $\begin{array}{l}\text { Day of ICU } \\
\text { administration }\end{array}$ & $\begin{array}{l}\text { BUD } 0.25 \mathrm{mg} / \mathrm{kg}+\text { PS } 100 \mathrm{mg} / \mathrm{kg}(\mathrm{NEB} \text {, } \\
\text { q8h) }\end{array}$ & PS 100 mg/kg ia, Q8h & 40 & 40 \\
\hline $\mathrm{Ke}, 2016^{[22]}$ & $\begin{array}{l}\text { GA }<32 \text { w, BW }<1500 \\
\text { g, RDS }\end{array}$ & $4 \mathrm{~h}$ after birth & $\begin{array}{l}\text { G1: BUD (pMDI } 0.25 \mathrm{mg} / \mathrm{kg} \text { every day until } \\
\text { ventilator withdraw }+ \text { PS } 200 \mathrm{mg} / \mathrm{kg} \text { ia) } \\
\text { G2: BUD }(0.25 \mathrm{mg} / \mathrm{kg})+\text { PS } 200 \mathrm{mg} / \mathrm{kg} \text { ia }\end{array}$ & $1 \mathrm{PS} 200 \mathrm{mg} / \mathrm{kg}$ ia & $\begin{array}{l}\text { G1: } 46 \\
\text { G2: } 46\end{array}$ & 46 \\
\hline Pan, $2017^{[23]}$ & $\begin{array}{l}\text { GA }<32 \text { w, BW }<1500 \\
\text { g, RDS with II history }\end{array}$ & $4 \mathrm{~h}$ after birth & BUD $0.2 \mathrm{mg} / \mathrm{kg}+\mathrm{PS} 70 \mathrm{mg} / \mathrm{kg}$ ia & PS $70 \mathrm{mg} / \mathrm{kg}$ ia & 15 & 15 \\
\hline Dg, 2017 $7^{[24]}$ & $\begin{array}{l}\mathrm{GA}<37 \mathrm{w}, \\
\mathrm{BW}<1500 \mathrm{~g}, \mathrm{RDS}\end{array}$ & $8 \mathrm{~h}$ after birth & $\begin{array}{l}\mathrm{GA}<37 \mathrm{w}, \\
\text { BUD } 0.25 \mathrm{mg} / \mathrm{kg}+\mathrm{PS} 150 \mathrm{mg} / \mathrm{kg} \text { ia }\end{array}$ & PS $150 \mathrm{mg} / \mathrm{kg}$ ia & 18 & 28 \\
\hline
\end{tabular}

GA: gestational age; BW: birth weight; RDS: respiratory distress syndrome; BUD: budesonide; BDP: beclomethasone propionate; PS: pulmonary surfactant; ia: via intratracheal administration; NEB: via nebulizer; pMDI: via pressure metered dose inhaler; G1: group 1; G2: group 2; II: intrauterine infection 
Table 2 GRADE profile of selected studies

\begin{tabular}{|c|c|c|c|c|c|c|c|}
\hline \multirow{2}{*}{ Study } & \multirow{2}{*}{ Design } & \multicolumn{6}{|c|}{ Quality assessment } \\
\hline & & Risk of bias & Inconsistency & Indirectness & Imprecision & Others & Quality \\
\hline Kuo, 2010 ${ }^{[16]} ;$ Yeh, 2008 $8^{[17]}$ & RCT & Not serious & Not serious & Not serious & Not serious & NA & High \\
\hline Sadeghnia, $2018^{[18]}$ & $\mathrm{RCT}$ & Serious $^{1}$ & Not serious & Not serious & Not serious & NA & Moderate \\
\hline Yeh, 2016 $6^{[19]}$ & RCT & Not serious & Not serious & Not serious & Not serious & NA & High \\
\hline Zimmeman, $2000^{[20]}$ & RCT & Not serious & Not serious & Not serious & Not serious & NA & High \\
\hline Cao, $2018^{[21]}$ & RCT & Not serious & Not serious & Not serious & Not serious & NA & High \\
\hline $\mathrm{Ke}, 2016^{[22]}$ & $\mathrm{RCT}$ & Serious $^{2}$ & Not serious & Not serious & Not serious & NA & Moderate \\
\hline Pan, 2017 $7^{[23]}$ & $\mathrm{RCT}$ & Serious $^{2}$ & Not serious & Not serious & Not serious & NA & Moderate \\
\hline Dg, $2017^{[24]}$ & RCT & Serious $^{2}$ & Not serious & Not serious & Not serious & NA & Moderate \\
\hline
\end{tabular}

${ }^{1}$ Interventions were not set as blinded to treatment practitioners; ${ }^{2}$ not describing the implementation of random sequence generation and allocation concealment

Table 3 Summary of findings through meta-analysis

\begin{tabular}{lll}
\hline \multirow{2}{*}{ Number of trials } & \multicolumn{2}{c}{ Effect (95\% CI) } \\
\cline { 2 - 3 } & Outcome & Estimate effect \\
\hline 8 & BPD incidence & RR=0.56 (95\% CI: 0.42-0.76) \\
6 & Mortality & RR=0.67 (95\% CI: 0.45-0.99) \\
5 & Percentage of infants using PS more than one time & RR=0.55 (95\% CI: 0.45-0.67) \\
2 & Incidence of infection & RR=0.95 (95\% CI: 0.59-1.52) \\
3 & Incidence of retinopathy & RR=0.92 (95\% CI: 0.62-1.38) \\
6 & Incidence of neuro-motor system impairment & RR=1.13 (95\% CI: $0.92-1.39)$ \\
\hline
\end{tabular}

that the incidence of BPD was significantly lower in both ICS intratracheal instillation subgroup and ICS inhalation subgroup, with RR 0.58 (95\% CI: $0.41-$ 0.82 ) and RR 0.47 (95\% CI: 0.24-0.95), respectively (fig. 2).

2.3.3 Mortality Six studies enrolling 608 patients reported mortality data. Meta-analysis of the fixed effects model indicated that the ICS group was associated with a lower mortality incidence than the placebo control group ( $\mathrm{RR}=0.67$; 95\% CI: 0.45-0.99, $P=0.04)$. Subgroup analysis showed that mortality was significantly lower only in ICS intratracheal instillation subgroup, not in ICS inhalation subgroup, with corresponding $\mathrm{RR}=0.64$ (95\% CI: $0.41-0.99, P=0.04)$ and $\mathrm{RR}=0.81$ (95\% CI: 0.34-1.94, $P=0.64$ ) (fig. 3).

\subsubsection{Percentage of Infants Using PS More Than}

One Time Five studies enrolling 681 patients analyzed the percentage of infants using PS more than one time. Meta-analysis of the random effects model indicated that the ICS group was associated with a lower percentage of infants using PS more than one time than the placebo control group $(\mathrm{RR}=0.551 ; 95 \%$ CI: $0.45-0.67, P<0.00001)$. Subgroup analysis showed the percentage of infants using PS more than one time was significantly lower only in ICS intratracheal instillation subgroup, not in ICS inhalation subgroup, with corresponding $\mathrm{RR}=0.56 \quad(95 \% \mathrm{CI}: 0.45-0.69$, $P<0.00001)$ and $\mathrm{RR}=0.35$ (95\% CI: $0.08-1.52, P=0.16)$ (fig. 4).

2.3.5 Adverse Events There was no significant difference in the incidence of infection or retinopathy of prematurity and neuro-motor system impairment between ICS and placebo control groups, with the corresponding RR 0.95 (95\% CI: 0.59-1.52), 0.92 (95\% CI: $0.62-1.38$ ) and 1.13 (95\% CI: 0.92-1.39), respectively.

\section{DISCUSSION}

Through comprehensive search and strictly screening, this meta-analysis includes 8 RCTs (9 articles), 4 studies (5 articles) of which followed the CONSORT guidance ${ }^{[25]}$ in the design, randomization and blind implementation, with a lower risk of selection bias. All the 8 studies reported the diagnostic criteria for NRDS or RDS and complementary follow-up process, with good control of selection bias and follow-up bias. This meta-analysis defines preterm infants with NRDS as the intervention population, within 1 day after birth as intervention initiation time, and presents the GRADE evaluation of evidence, helps to aid healthcare professionals for clinical decisions. However, subtle underlying bias of the studies included in the review remains a possible limitation, as in any other systematic review although we excluded the studies with high risk of bias.

With the establishment of NICU, through the application of PS, the implementation of pulmonary ventilation protection strategy and the use of glucocorticoids before delivery, the survival rate of premature infants with very low and ultra-low birth weight improved, but the incidence of BPD was increasing accordingly, which severely affected the prognosis as a chronic respiratory disease in premature 


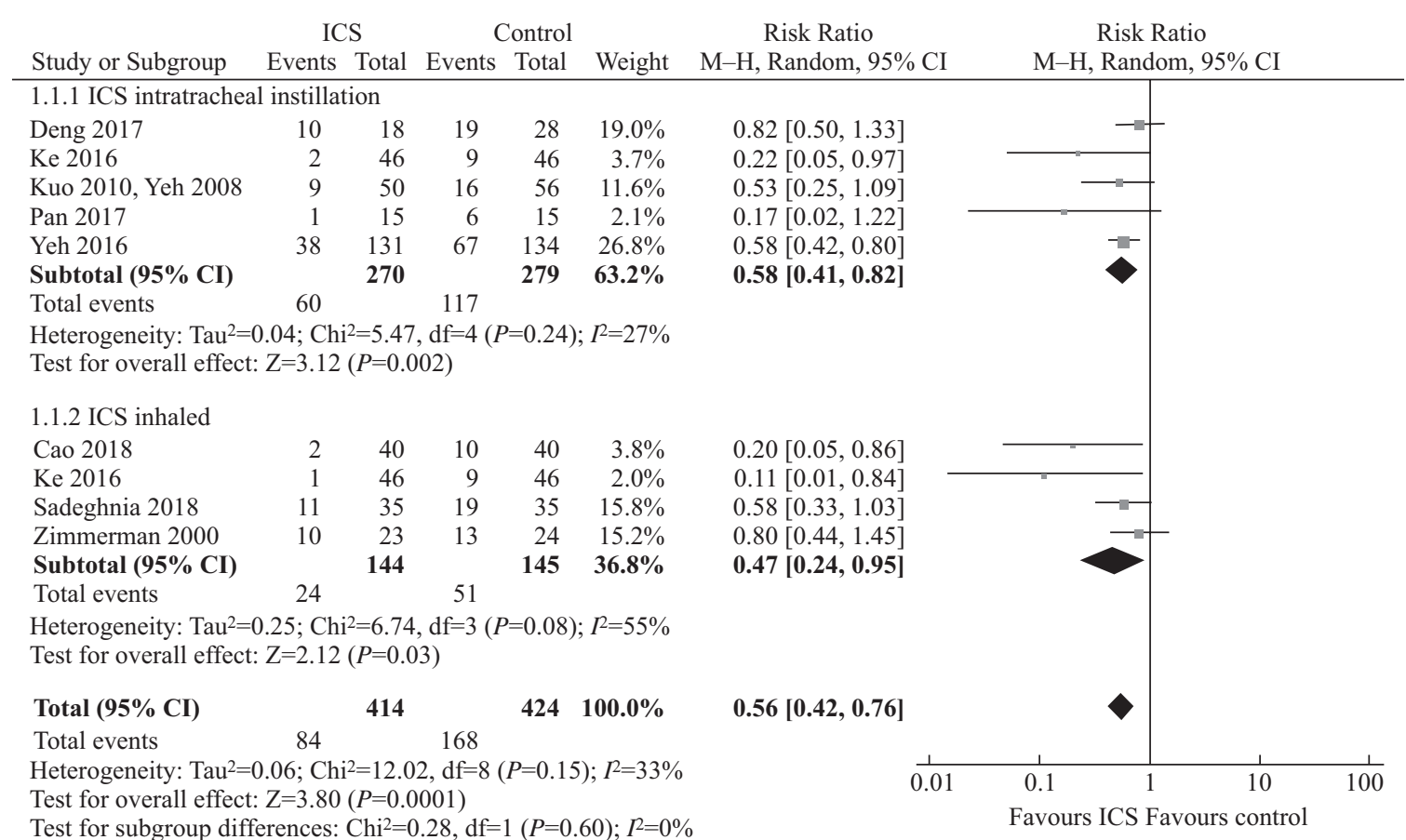

Fig. 2 Meta-analysis of the incidence of BPD in ICS group and placebo control group

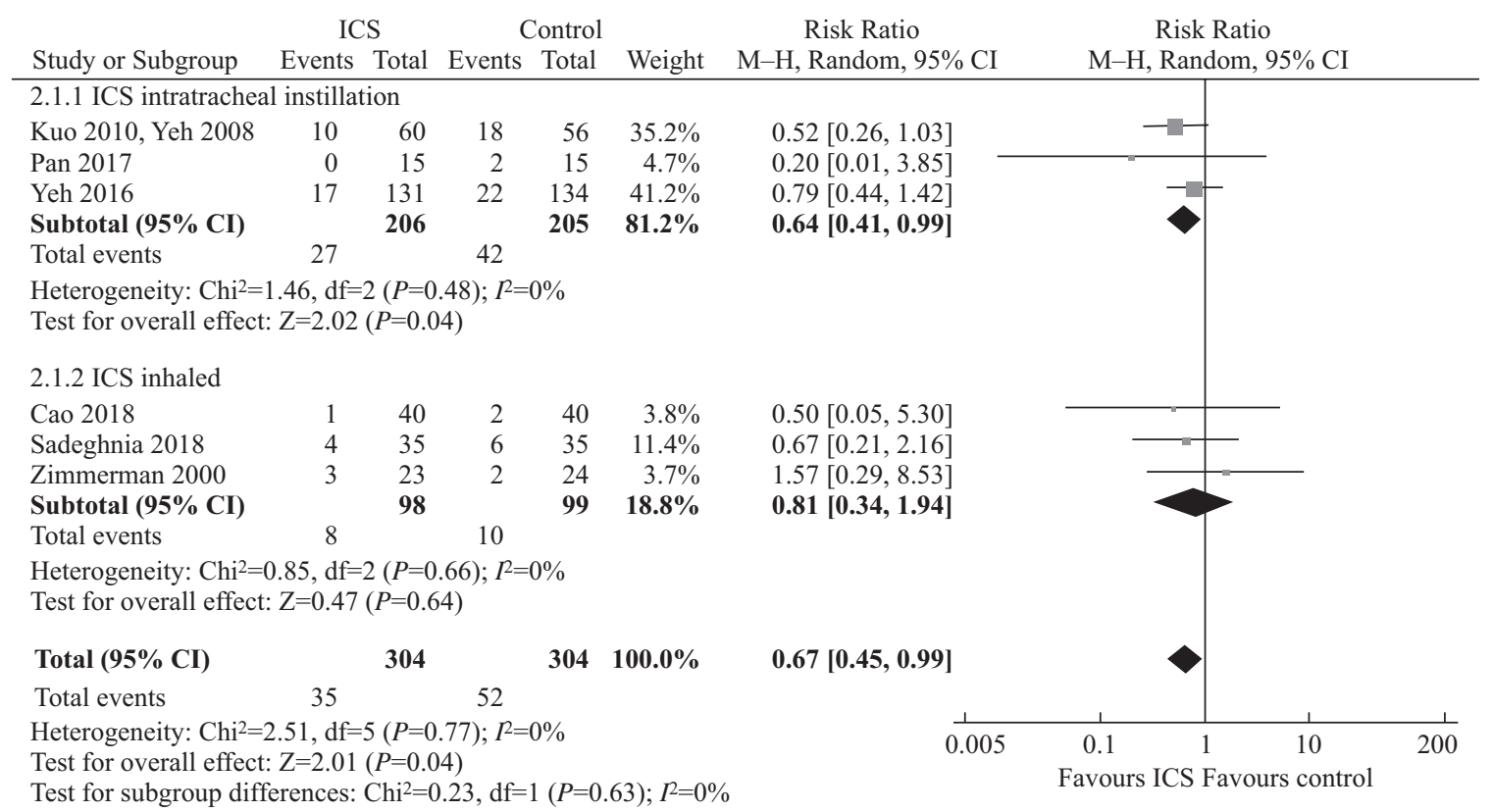

Fig. 3 Meta-analysis of mortality comparison between ICS group and placebo control group

infants. The current treatments of BPD mostly focus on improving the symptoms of children, which cannot fundamentally improve the lung damage that has already occurred. Therefore, it is necessary to find effective and available preventive strategy to reduce the occurrence of $\mathrm{BPD}^{[26]}$.

Cohort studies have uncovered risk factor of BPD, such as gestational age, lower birth weight, NRDS and mechanical ventilation history ${ }^{[27]}$. Thus, focus on the treatment of preterm infants with NRDS, is the priority of preventive strategy to reduce the occurrence of BPD. Inflammatory injury plays a key role in BPD pathogenesis. ICS such as budesonide showed local high-efficient anti-inflammatory effects in the airway. Directly targeting the airway and lungs through aerosol inhalation by nebulizer or pMDI and intratracheal instillation, ICS can block lung inflammation process and reduce inflammation damage to lung tissue, inhibit 


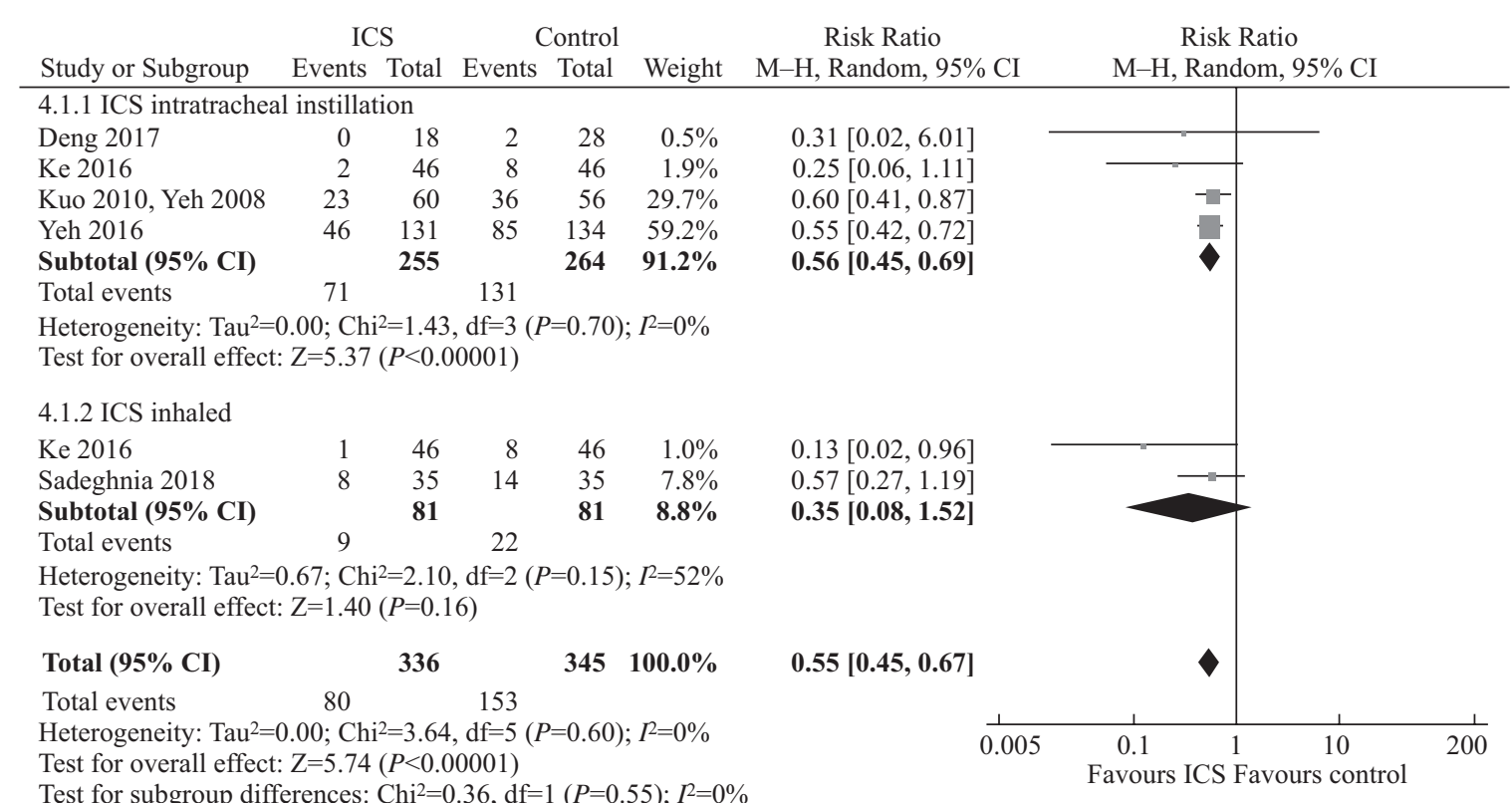

Fig. 4 Meta-analysis of rate of additional PS usage in ICS group and placebo control group

excessive abnormal repair of lung tissue, increase lung compliance, improve lung ventilation outcome ${ }^{[28]}$, and shorten the mechanical ventilation time, thus reduce the incidence of BPD. PS is routinely used in children with NRDS to promote normal alveolar recovery, stabilize the terminal airway, and reduce atelectasis. PS can diffuse rapidly under the gradient through the lung surface and help other drugs diffuse to the lungs. Yeh's study ${ }^{[6]}$ confirmed that a mixture of PS and budesonide suspension not only improved drug delivery but also had a synergistic clinical benefit. The results of our meta-analysis showed that the combined application of glucocorticoids and PS in the airway after NRDS can reduce the occurrence of BPD and mortality incidence in premature infants, and reduce the likehood of using PS more than one time, which is consistent with the published meta-analysis ${ }^{[29-32]}$. In this meta-analysis, we further found that the combination of ICS and PS by intratracheal instillation can reduce the mortality of BPD in premature infants with confirmed NRDS. These patients are high-risk groups with BPD, so the ICS and PS by intratracheal instillation intervention has the highest benefit/risk ratio. ICS intratracheal instillation can also reduce the percentage of infants using PS more than one time, and shows good pharmacoeconomic value. The underlying reason may be ICS improving lung compliance and ventilatory function and promoting alveolar type II cell synthesis and secretion ${ }^{[33]}$. Subgroup analysis showed that ICS intratracheal instillation in combination with PS might be superior to ICS via nebulizer/pMDI inhalation, which is contrary to Ke's findings[ ${ }^{[22]}$, and this may be related to the limited number of samples and the heterogeneity between the studies. The merits of these two methods of ICS administration still require a large sample size RCT to verify.

This meta-analysis shows that there is no statistically significant difference in incidence of infection and ROP between the ICS group and the placebo control group. The meta-analysis also investigated the risk of adverse neurological events, including recent complications such as intraventricular hemorrhage and cerebral palsy and long-term complications such as neuro-motor system dysplasia of 3 years of follow-up. All data suggest that short-term use of ICS does not increase the risk of infection, ROP and neuro-motor system impairment.

To sum up, according to the meta review of enrolled 8 studies, early administration of ICS and PS is an effective and safe option for preterm infants with NRDS in preventing BPD and reducing mortality, decreasing the additional PS usage, especially for the ICS intratracheal instillation subgroup. Furthermore, the appropriate dose and duration of ICS, combined use of inhalation or instillation of ICS with PS and the long-term safety of airway administration of corticosteroids need to be assessed in large trials.

\section{Conflict of Interest Statement}

The authors declare that they have no conflicts of interest or financial disclosures to report.

\section{REFERENCES}

1 Carvalho CG, Silveira RC, Procianoy RS. Ventilatorinduced lung injury in preterm infants. Revista Brasileira De Terapia Intensiva, 2013,25(4):319-326

2 Yeh TF, Lin YJ, Hsieh WS, et al. Early postnatal dexamethasone therapy for the prevention of chronic 
lung disease in preterm infants with respiratory distress syndrome: a multicenter clinical trial. Pediatrics, 1997,100(4):e3-e3

3 Collaborative Dexamethasone Trial Group. Dexamethasone therapy in neonatal chronic lung disease: an international placebo-controlled trial. Pediatrics, 1991,88(3):421-427

4 Cummings JJ, D’Eugenio DB, Gross SJ. A controlled trial of dexamethasone in preterm infants at high risk for bronchopulmonary dysplasia. N Engl J Med, 1989,320(23):1505-1510

5 Vermont Oxford Network Steroid Study Group. Early postnatal dexamethasone therapy for the prevention of chronic lung disease. Pediatrics, 2001,108(3):741-748

6 Yeh TF, Lin YJ, Huang CC, et al. Early dexamethasone therapy in preterm infants: a follow-up study. Pediatrics, 1998,101(5):E7

7 Avery GB, Fletcher AB, Kaplan M, et al. Controlled trial of dexamethasone in respirator-dependent infants with bronchopulmonary dysplasia. Pediatrics, 1985,75(1):106-111

8 Yeh TF, Lin YJ, Lin HC, et al. Outcome at school age after postnatal dexamethasone therapy for lung disease of prematurity. N Engl J Med, 2004,350(13):1304-1313

9 Grigg J, Arnon S, Jones T, et al. Delivery of therapeutic aerosols to intubated babies. Arch Dis Child, 1992,67(1):25-30

10 Cole $\mathrm{CH}$, Colton $\mathrm{T}$, Shah BL, et al. Early inhaled glucocorticoid therapy to prevent bronchopulmonary dysplasia. N Engl J Med, 1999,340(13):1005-1010

11 Fujiwara T, Chida S, Watabe Y, et al. Artificial surfactant therapy in hyaline membrane disease. Lancet, 1980,1(8159):55-59

12 Ferrara TB, Hoekstra RE, Johnson P, et al. Localization of surfactant in neonatal lung after exogenous administration. J Pediatr, 1987,111(3):463-466

13 Tam D, Von Arnim V, Mckinley GH, et al. Marangoni convection in droplets on superhydrophobic surfaces. J Fluid Mechan, 2009,624:101

14 Fajardo C, Levin D, Garcia M, et al. Surfactant versus saline as a vehicle for corticosteroid delivery to the lungs of ventilated rabbits. Pediatr Res, 1998,43(4):542547

15 Kharasch VS, Sweeney TD, Fredberg J, et al. Pulmonary surfactant as a vehicle for intratracheal delivery of technetium sulfur colloid and pentamidine in hamster lungs. Am Rev Respir Dis, 1991,144(4):909-913

16 Kuo HT, Lin HC, Tsai CH, et al. A follow-up study of preterm infants given budesonide using surfactant as a vehicle to prevent chronic lung disease in preterm infants. J Pediatr, 2010,156(4),537-541.

17 Yeh TF, Lin HC, Chang CH, et al. Early intratracheal instillation of budesonide using surfactant as a vehicle to prevent chronic lung disease in preterm infants: a pilot study. Pediatrics, 2008,121(5): e1310-e1318

18 Sadeghnia A, Beheshti BK, Mohammadizadeh M. The effect of inhaled budesonide on the prevention of chronic lung disease in premature neonates with respiratory distress syndrome. Int J Prev Med, 2018,9:15

19 Yeh TF, Chen CM, Wu SY, et al. Intratracheal administration of budesonide/surfactant to prevent bronchopulmonary dysplasia. Am J Respirat Crit Care
Med, 2016,193(1):86-95

20 Zimmerman JJ, Gabbert D, Shivpuri C, et al. Meterdosed, inhaled beclomethasone initiated at birth to prevent bronchopulmonary dysplasia. Pediatr Crit Care Med, 2000,1(2):140-145

21 Cao YY, Yao G, Wang Y, et al. Aerosol Inhalation of Budesonide and Pulmonary Surfactant to Prevent Bronchopulmonary Dysplasia. J Pediatr Pharmacy, 2018,24(03):25-29

$22 \mathrm{Ke} \mathrm{H}$, Li ZK, Yu XP, et al. Efficacy of different preparations of budesonide combined with pulmonary surfactant in the treatment of neonatal respiratory distress syndrome: a comparative analysis. Zhongguo Dang Dai Er Ke Za Zhi (Chinese), 2016,18(5):400-404

23 Pan J, Chen MW, Ni WQ, et al. Clinical efficacy of pulmonary surfactant combined with budesonide for preventing bronchopulmonary dysplasia in very low birth weight infants. Zhongguo Dang Dai Er Ke Za Zhi (Chinese), 2017,19(2):137-141

24 Deng LJ, Peng HB, Gong XQ. Effect of budesonide combined with pulmonary surfactant on severe respiratory distress syndrome in bronchopulmonary dysplasia. Chin J Neonatol (Chinese), 2017,32(5):361364

25 Schulz KF, Altman DG, Moher D. CONSORT 2010 statement: updated guidelines for reporting parallel group randomised trials. BMC Med, 2010,8(1):18

26 Bhandari A, Panitch H. An update on the post-NICU discharge management of bronchopulmonary dysplasia. Semin Perinatol, 2018,42(7):471-477

27 Nagiub M, Kanaan U, Simon D, et al. Risk factors for development of pulmonary hypertension in infants with bronchopulmonary dysplasia: systematic review and meta-analysis. Paediatr Respirat Rev, 2017,23:27-32

28 Merz U, Kusenbach G, Häusler M, et al. Inhaled budesonide in ventilator-dependent preterm infants: a randomized, double-blind pilot study. Neonatology, 1999,75(1):46-53

29 Onland W, Offringa M, van Kaam A. Late ( $\geq 7$ days) inhalation corticosteroids to reduce bronchopulmonary dysplasia in preterm infants. Cochrane Database Syst Rev, 2012,4:CD2311

30 Venkataraman R, Kamaluddeen M, Hasan SU, et al. Intratracheal administration of budesonide-surfactant in prevention of bronchopulmonary dysplasia in very low birth weight infants: a systematic review and metaanalysis. Pediatr Pulmonol, 2017,52(7):968-975

31 Zhang ZQ, Zhong Y, Huang XM, et al. Airway administration of corticosteroids for prevention of bronchopulmonary dysplasia in premature infants: a meta-analysis with trial sequential analysis. BMC Pulmon Med, 2017,17(1):207

32 Zeng L, Tian J, Song F, et al. Corticosteroids for the prevention of bronchopulmonary dysplasia in preterm infants: a network meta-analysis. Arch Dis Child Fetal Neonatal Ed, 2018,103(6):F506-F511

33 Yu ZW, Zhang JH. Effect of inhaled budesonide on surfactant protein expression in asthmatic mice. Allergy Asthma Proc. 2008,29(5):486-492

(Received Oct. 28, 2018; revised Mar. 7, 2019) 